\title{
Formation of c-axis Aligned Polycrystal Hydroxyapatite Using High Magnetic Field with Mechanical Sample Rotation
}

\author{
Jun Akiyama ${ }^{1, *}$, Masami Hashimoto ${ }^{2}$, Hiroaki Takadama ${ }^{2}$, Fukue Nagata $^{3}$, \\ Yoshiyuki Yokogawa ${ }^{3}$, Kensuke Sassa ${ }^{1}$, Kazuhiko Iwai ${ }^{1}$ and Shigeo Asai ${ }^{1}$ \\ ${ }^{1}$ Department of Materials, Physics and Energy Engineering, Nagoya University, Nagoya 464-8603, Japan \\ ${ }^{2}$ Japan Fine Ceramics Center, Nagoya 456-8587, Japan \\ ${ }^{3}$ National Institute of Advanced Industrial Science and Technology, Nagoya 463-8560, Japan
}

\begin{abstract}
It is possible to control the crystal alignment of non-magnetic materials such as anisotropic ceramics and polymeric materials using a high magnetic field. However, the alignment of the c-axis direction in hexagonal crystals, with a magnetic susceptibility of $\chi_{\mathrm{c}}<\chi_{\mathrm{a}, \mathrm{b}}$, is uncontrollable using a static magnetic field, because the c-axis can rotate in the plane perpendicular to the direction of the magnetic field. In this study, a high magnetic field and mechanical rotation are simultaneously imposed on a sample in order to align crystals parallel to that axis (c-axis) which has small magnetic susceptibility. This process of alignment was applied to hydroxyapatite (HAp) crystals and a sample was obtained, in which the c-axis of the HAp crystals aligned in a particular direction.
\end{abstract}

(Received October 26, 2004; Accepted January 11, 2005)

Keywords: hydroxyapatite, crystal alignment, high magnetic field, slip casting

\section{Introduction}

Hydroxyapatite (HAp), which is a main component of bones and teeth in vertebrates, is one type of bioactive ceramic. It has been widely applied as a biomaterial for use as a bone filler, artificial dental root and a synthetic blood vessel. HAp is also a useful adsorbent material in liquid chromatography, because of its high sorbability for proteins. Different kinds of functional groups in proteins are adsorbed on different planes of HAp crystals, because HAp exhibits anisotropic specific adsorption, caused by its hexagonal crystal structure. For example, the c-plane of HAp is the adsorption site for amino acids in a protein and for sodium ions in a buffer solution, where phosphoric acid is the main occupant in this crystal plane. ${ }^{1)}$ On the other hand, the a,bplanes are the favored adsorption sites for carboxyl groups in a protein, and for the phosphoric acid ion in a buffer solution, because calcium is the main occupant in the crystal plane. ${ }^{1)}$ This anisotropic nature is useful for the improvement of sorbability and bioactivity on the surface of biomaterials and adsorbents. ${ }^{2)}$ Therefore, the alignment of HAp crystals, in a particular direction, imparts specific functionality to this material.

The crystal alignment of HAp has been investigated using various methods, such as slip casting under a high magnetic field, ${ }^{3)}$ self-organization between HAp and collagen, ${ }^{4)}$ a hydrolysis reaction process $^{5)}$ and pulse current pressure sintering. ${ }^{6)}$ Some of these processes are complicated or it is not easy to obtain a bulk sample in which the c-axis of HAp crystals is aligned in the same direction. On the other hand, it was reported that uni-directional alignment of a polymeric material with a magnetic susceptibility of $\chi_{\mathrm{c}}<\chi_{\mathrm{a}, \mathrm{b}}$ was successfully obtained by rotating the magnetic field generated by permanent magnets. ${ }^{7)}$ However, it is difficult to adopt this process for the alignment of crystals which have a small magnetic anisotropy, because the maximum magnitude of the

*Graduate Student, Nagoya University magnetic field generated by a permanent magnet is in the order of 1 tesla.

In this study, a high static magnetic field and mechanical rotation was simultaneously applied during a slip casting process, to induce the c-axis alignment of HAp poly crystals, and the degree of crystal alignment was then examined by $\mathrm{X}$-ray diffraction (XRD).

\section{Principle of Crystal Alignment}

In the field of materials science and technology, it has been found that a high magnetic field has the ability to control crystal alignment of not only magnetic materials, but also non-magnetic materials. ${ }^{8-10)}$ The principle of crystal alignment of a non-magnetic material in a high magnetic field is explained from a perspective of magnetization energy. When a non-magnetic material is submerged in a magnetic field, the magnetization energy per unit volume, $U$, is approximately indicated as

$$
U \cong-\frac{\mu_{0} \chi H^{2}}{2}
$$

where $\mu_{0}$ is the magnetic permeability in a vacuum, $\chi$ is the magnetic susceptibility parallel to the magnetic field, and $H$ is the intensity of the external magnetic field.

If the crystal structure has a magnetic anisotropy, the magnetic energy also has an anisotropic nature. The magnetization energy for hexagonal crystals is expressed as eq. (2) in the case that the magnetic field is parallel to the c-axis, and as eq. (3) in the case that the magnetic field is parallel to the a,b-axis.

$$
\begin{gathered}
U_{\mathrm{c}} \cong-\frac{\mu_{0} \chi_{\mathrm{c}} H^{2}}{2} \\
U_{\mathrm{a}, \mathrm{b}} \cong-\frac{\mu_{0} \chi_{\mathrm{a}, \mathrm{b}} H^{2}}{2}
\end{gathered}
$$

where $\mathrm{a}, \mathrm{b}$ and $\mathrm{c}$ indicate the crystallographic axes. If a crystal is set in a magnetic field, then the crystal rotates in 
order to reduce the magnetization energy, so as to be in a stable conformation. For a magnetically anisotropic crystal with the relation that $\chi_{\mathrm{c}}>\chi_{\mathrm{a}, \mathrm{b}}$, the magnetization energy becomes the smallest when the c-axis of the crystal is parallel to the direction of the magnetic field $\left(U_{\mathrm{a}, \mathrm{b}}>U_{\mathrm{c}}\right)$. Therefore, the c-axis of the crystal aligns parallel to the direction of the magnetic field, in order to minimize the magnetization energy. On the other hand, the a,b-axis of the crystal aligns parallel to the direction of the magnetic field for those crystals with the relation $\chi_{\mathrm{c}}<\chi_{\mathrm{a}, \mathrm{b}}\left(U_{\mathrm{a}, \mathrm{b}}<U_{\mathrm{c}}\right)$. For the crystal alignment of HAp using a high magnetic field, it is experimentally observed that the a,b-axis of the crystals aligns parallel to the direction of the magnetic field. Therefore, the magnetic susceptibility of the HAp crystal is expected to be $\chi_{\mathrm{c}}<\chi_{\mathrm{a}, \mathrm{b}}$, considering the magnetization energy and the experimental result. These facts indicate that it is difficult to control the c-axis alignment of HAp crystals using a static magnetic field. The relationship between the imposed magnetic field direction and the direction of a hexagonal crystal with magnetic anisotropy, $\chi_{\mathrm{c}}<\chi_{\mathrm{a}, \mathrm{b}}$, is summarized in Fig. 1.

The crystal direction with no magnetic field is random, as shown in Fig. 1(a). If a magnetic field is imposed on the crystals, the a,b-axis of each crystal aligns parallel to the direction of the magnetic field, as shown in Fig. 1(b), because the magnetic susceptibility of the a,b-axis is larger than that of the c-axis. Only the a,b-axis is observed in the plane perpendicular to the direction of the magnetic field, while all of the axes are observed in the plane parallel to the direction of the magnetic field. That is, c-axis alignment is impossible by imposing a static magnetic field.

Therefore, a new method is required for crystal alignment in the c-axis direction, in order to utilize the anisotropic nature of crystals for industrial applications. A new method is proposed for the c-axis alignment of a hexagonal crystal with small magnetic anisotropy of the following relation, $\chi_{\mathrm{c}}<\chi_{\mathrm{a}, \mathrm{b}}$, based on the following principle. If a magnetic field is imposed on a crystal in the $x$-direction, the c-axis of the crystal rotates to be in the $y-z$ plane, since it is the most stable condition in consideration of the magnetization energy. On the other hand, if a magnetic field in the $y$-direction is imposed on the crystal, the c-axis of the crystal rotates to be in the $\mathrm{x}-\mathrm{z}$ plane. Therefore, by rotating the sample in the $x-y$ plane of the static high magnetic field, in order to change the imposing magnetic field direction, it is expected that the c-axis aligns parallel to the z-axis, which is a stable position for a magnetic field in the $x-y$ plane. (Fig. 2)

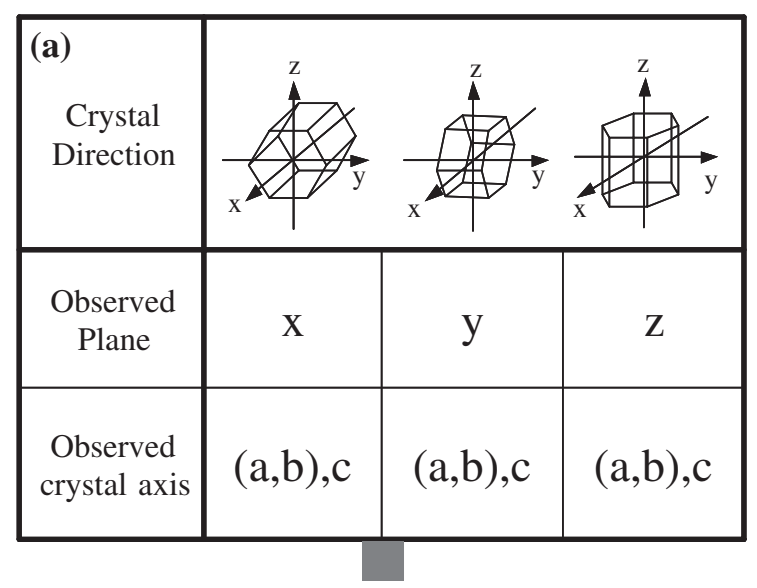

Imposition of static magnetic field in $\mathrm{z}$ direction

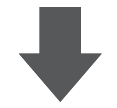

\begin{tabular}{|c|c|c|c|}
\hline $\begin{array}{c}\text { Crystal } \\
\text { Direction }\end{array}$ & & \\
\hline $\begin{array}{c}\text { Observed } \\
\text { Plane }\end{array}$ & $\mathrm{x}$ & $\mathrm{y}$ & $\mathrm{z}$ \\
\hline $\begin{array}{c}\text { Observed } \\
\text { crystal axis }\end{array}$ & $(\mathrm{a}, \mathrm{b}), \mathrm{c}$ & $(\mathrm{a}, \mathrm{b}), \mathrm{c}$ & $a, b$ \\
\hline
\end{tabular}

Fig. 1 Alignment of hexagonal crystal with the relation $\chi_{\mathrm{c}}<\chi_{\mathrm{a}, \mathrm{b}}$ by static magnetic field.

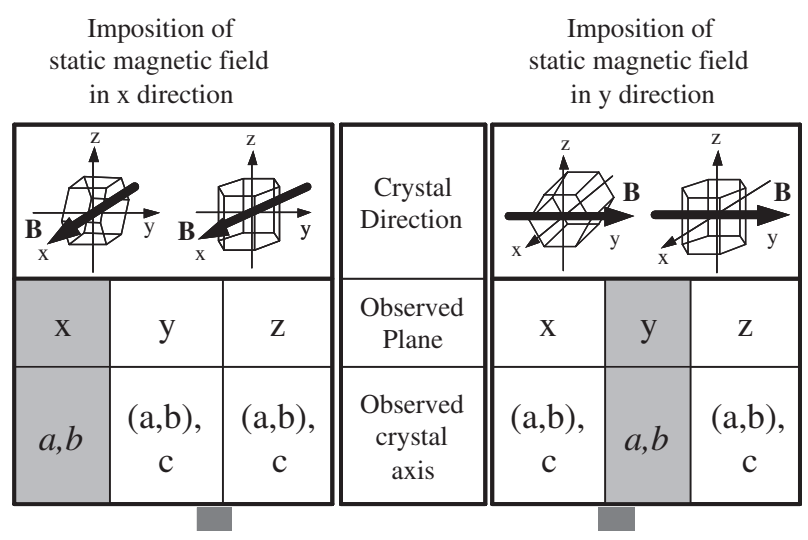

Changing the imposing direction of high magnetic field in $\mathrm{x}-\mathrm{y}$ direction by sample rotation

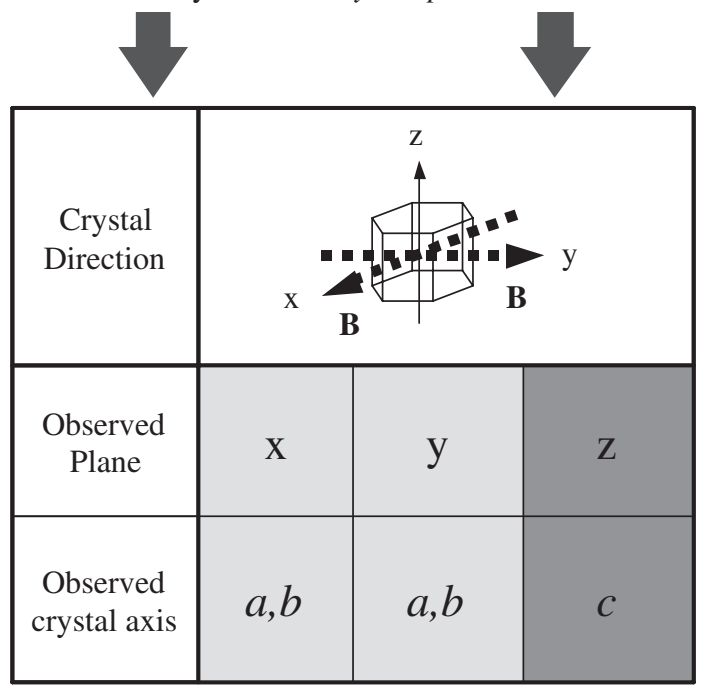

Fig. 2 C-plane alignment of hexagonal crystal with the relation by $\chi_{\mathrm{c}}<\chi_{\mathrm{a}, \mathrm{b}}$ changing the imposing direction of static magnetic field. 


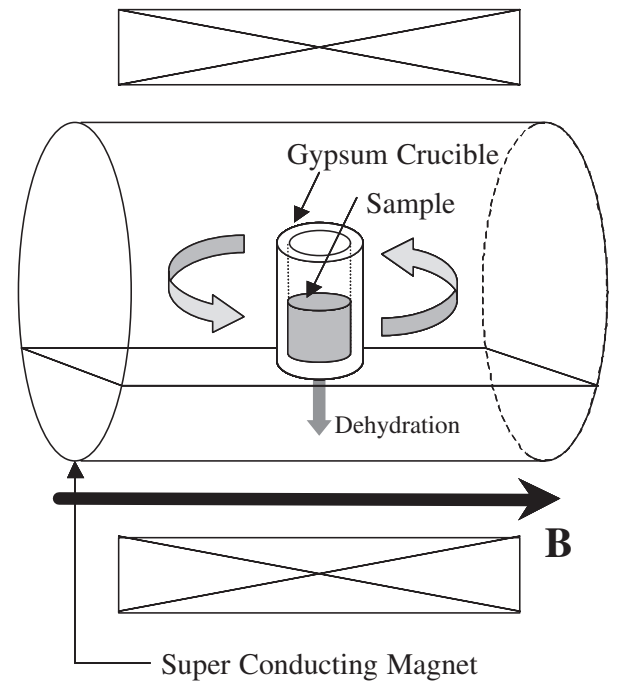

Fig. 3 Experimental apparatus.

\section{Experimental}

The experimental apparatus is shown in Fig. 3. A slurry containing HAp particles (Taihei Chemical Co., mean diameter: $2.17 \mu \mathrm{m}$ ) and distilled water was mixed with a deflocculant, and the mixture was mechanically ground in a mortar for 4 hours, to reduce the aggregate particles into single crystals. The slurry was then poured into a gypsum crucible (I.D. $22 \mathrm{~mm}$, volume $1.5 \times 10^{3} \mathrm{~mm}^{3}$ ), and set in the bore of a super conducting magnet for slip casting with or without sample rotation. In order to align the c-axis of each HAp crystal parallel to the gravitational direction in the slip casting process, the sample was rotated with $0.3 \mathrm{rad} / \mathrm{s}$ in the horizontal plane under the imposition of a horizontal $10 \mathrm{~T}$ magnetic field. After slip casting, the sample was dried in air for 24 hours without a magnetic field, and was sintered at $1423 \mathrm{~K}$ for 2 hours in air using an electric furnace.

A relative facial angle, $\theta$, which is defined by eq. (4), is adopted to evaluate the mean degree of the crystal alignment of HAp from the results of XRD analysis for the upper surface of the sintered samples.

$$
\theta=\frac{\sum\left(I_{h_{\mathrm{i}} k_{\mathrm{i}} l_{\mathrm{i}}} \times \theta_{h_{\mathrm{i}} k_{\mathrm{i}} l_{\mathrm{i}}}\right)}{\sum I_{h_{\mathrm{i}} k_{\mathrm{i}} l_{\mathrm{i}}}}
$$

where $I_{h_{\mathrm{i}} k_{\mathrm{i}} l_{\mathrm{i}}}$ is the intensity of $\left(h_{\mathrm{i}} k_{\mathrm{i}} l_{\mathrm{i}}\right)$ in the observed XRD pattern, and $\theta_{h_{\mathrm{i}} k_{\mathrm{i}} l_{\mathrm{i}}}$ is the facial angle between the $\left(h_{\mathrm{i}} k_{\mathrm{i}} l_{\mathrm{i}}\right)$ plane and the c-plane. The relative facial angle, $\theta$, reduces to 0 degrees when the c-planes of all the crystals are aligned parallel to the measured surface, but becomes 90 degrees when the a,b-planes of all the crystals are parallel to the measured surface.

\section{Results and Discussion}

The XRD patterns of the sintered samples under the various experimental conditions are shown in Fig. 4. Many different peaks are observed in the XRD patterns of the sample without imposition of a magnetic field and sample rotation during the slip casting, as shown in Fig. 4(a). The

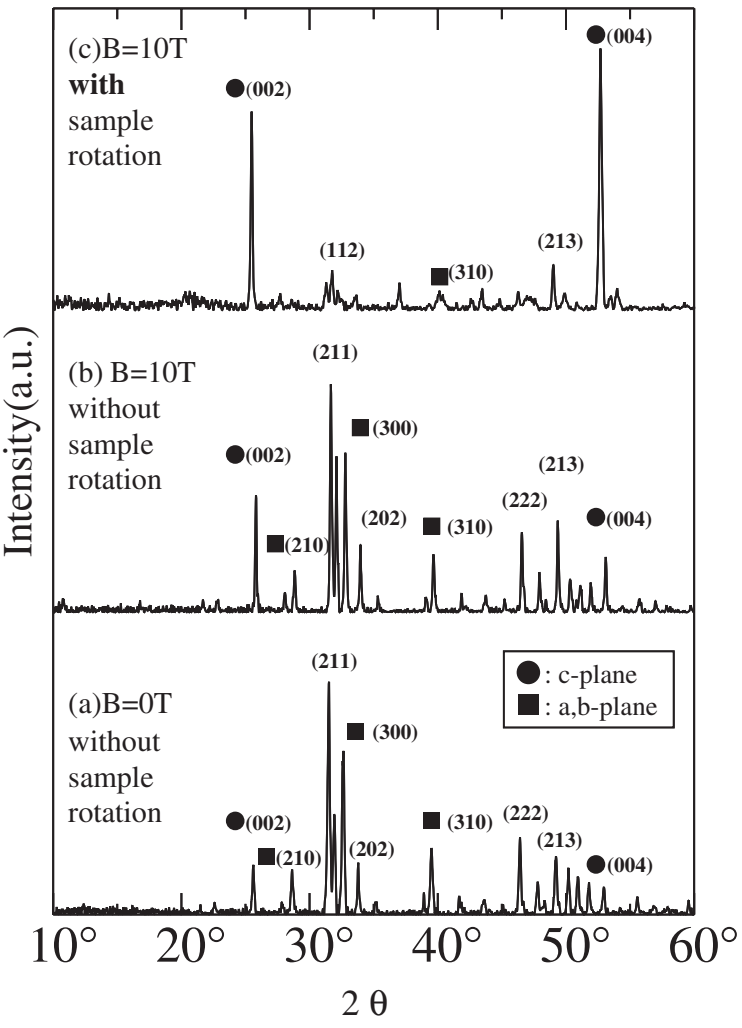

Fig. 4 XRD Analysis of sintered HAp samples.

relative facial angle of this sample is 60.2 degrees. The XRD pattern of the sample that received treatment under a $10 \mathrm{~T}$ magnetic field and without sample rotation during the slip casting is shown in Fig. 4(b). The relative facial angle of this sample is 53.6 degrees. This means that the c-plane at the upper surface of the sample is somewhat increased by the imposition of the magnetic field. However, the peaks of other planes still remain. This result agrees with the theoretical prediction that the complete c-plane alignment, for a sample with the physical property of $\chi_{\mathrm{c}}<\chi_{\mathrm{a}, \mathrm{b}}$, is difficult by imposition with a static magnetic field. The XRD pattern of the upper surface of the sample treated under a $10 \mathrm{~T}$ magnetic field and with sample rotation during the slip casting is shown in Fig. 4(c). Only the peaks corresponding to the c-plane, such as (002) and (004), still appear and other peaks almost disappear in the XRD pattern. The relative facial angle is considerably decreased to 23.0 degrees. That is, most of HAp crystals in this sample have aligned the c-plane parallel to the direction of gravity by sample rotation and imposition of a magnetic field during the slip casting process.

\section{Conclusion}

For the c-axis alignment of HAp crystals, a high static magnetic field and mechanical rotation of the sample have been applied during the slip casting process. The average facial angle of the sample after sintering was evaluated, and the sample treated with a magnetic field and mechanical rotation resulted in an average facial angle of 23.0 degrees, while that without treatment of the magnetic field and mechanical rotation was 60.2 degrees. Therefore, this hybrid 
process, encompassing both treatment with a magnetic field and mechanical rotation, is a useful tool for imposing the c-axis alignment of HAp.

\section{Acknowledgements}

This research was partially supported by the Ministry of Education, Culture, Sports, Science and Technology, Grantin-Aid for Exploratory Research, (No 16656209), "Creation of Nature-Guided Materials Processing" of the 21st Century COE Program and research support program of SEKISUI CHEMICAL Company.

\section{REFERENCES}

1) T. Kawasaki: J. Chromatography 151 (1978) 95-112.

2) T. Kawasaki, M. Nimura and Y. Kobayashi: J. of. Chromatography $\mathbf{5 1 5}$
(1990) 91-123.

3) K. Inoue, T. Marukawa, K. Sassa, Y. Yokogawa, Y. Sakka, M. Okido and S. Asai: Proc. 4th Int. Conf. on Electromagnetic Processing of Materials, Oct. 14-17 (2003) pp. 513-516.

4) M. Kikuchi, S. Itoh, S. Ichinose, K. Shinomiya and J. Tanaka: Biomaterials 22 (2001) 1705-1711.

5) K. Ohta, M. Kikuchi and J. Tanaka: Proc. The 25th Annual Meeting of the Japanese Society for Biomaterials Dec. 16-17 (2003) pp. 323.

6) Y. Watanabe, T. Ikoma, Y. Suetugu, J. Tanaka and Y. Moriyosi: Proc The 25th Annual Meeting of the Japanese Society for Biomaterials Dec. 16-17 (2003) pp. 325.

7) M. Yoshino, T. Kimura, T. Yamane and M. Yamato: Proc. 7th Symposium on New Magneto-Science, Nov. 5-17 (2003) pp. 69.

8) H. Morikawa, K. Sassa and S. Asai: Mater. Trans. 39 (1998) 814-818.

9) T. Suzuki, Y. Sakka and K. Kitazawa: J. Cera. Soc. JP 109 (2001) 886890.

10) S. Asai, K. Sassa and M. Tahashi: Sci. Technol. Adv. Mater. 4 (2003) 455-460. 